\title{
CONVERSION-SPACE TIME PROFILES OF STIRRED TANK REACTORS CONTINUOUSLY FED WITH REACTANTS AND CATALYST UNDER CONDITIONS OF STRONG CATALYST DEACTIVATION
}

\author{
E. FLASCHEL ${ }^{\dagger}$ \\ Technische Fakultät, Universität Bielefeld, P.O. Box 1001 31, D-33501 Bielefeld, Germany \\ A. MARGOT \\ Koreco Ltd., P.O. Box 12, CH-3510 Konolfingen, Switzerland \\ M. DOHMEN \\ Technische Fakultät, Universität Bielefeld, P.O. Box 1001 31, D-33501 Bielefeld, Germany \\ and \\ A. RENKEN \\ Institut de génie chimique, Ecole Polytechnique Fédérale de Lausanne, CH-1015 Lausanne, Switzerland
}

(Received 25 October 1993; accepted in revised form 8 September 1994)

\begin{abstract}
Feeding continuously operated stirred tank rectors with reactants and homogeneous catalysts subject to inactivation will usually lead to a limited substrate conversion with increasing space time. However, it is shown that a maximum of conversion may be observed under certain circumstances. Guided by experimental evidence, the theoretical background is discussed for identifying reaction systems for which such conversion maxima at distinct space times may be obtained. The analysis shows that this phenomenon may only be observed if the catalyst undergoes deactivation characterized by a kinetics of less than first order. Therefore, a likely case for experimental verification consists in an enzymic reaction accompanied by proteolytic degradation of the substrate transforming enzyme due to the presence of a protease.
\end{abstract}

\section{INTRODUCTION}

This communication aims at drawing attention to a special behaviour of continuous stirred tank reactors which, to the knowledge of the authors, has been overlooked so far. The phenomenon of interest is associated with the operation of stirred tank reactors continuously fed with reactants and catalyst, whereby the latter is not retained and undergoes strong deactivation. Interest in this question arose, when the partial hydrolysis of whey proteins by means of the soluble enzymic catalyst trypsin in continuously operated stirred tank reactors was studied (Margot, 1992). Under conditions of strong enzyme deactivation, a distinct maximum conversion occurred in the conversion (NPN: "non-protein nitrogen", solubilized fraction of proteins)-space time profile. For convenience, a typical graph is presented in Fig. 1. Details of the experimental work related to these data will be presented elsewhere.

The following discussion will focus on this strange behaviour characterized by a decline of substrate conversion with increasing space time. Because of the complexity of the experimental system studied by Margot (1992), the discussion will emphasize the theoretical background of this phenomenon.

'Author to whom correspondence should be addressed.

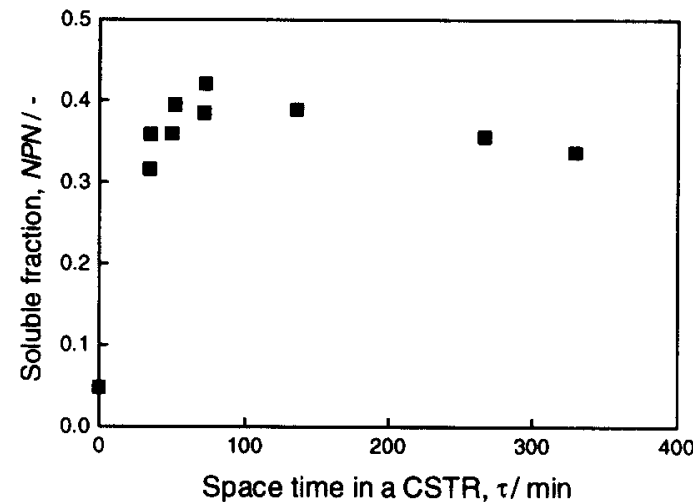

Fig. 1. Solubilization of whey proteins by soluble trypsin in a continuously operated stirred tank reactor (operating conditions: $S_{0}=20 \%(\mathrm{w} / \mathrm{w}), E_{0} / S_{0}=0.01 \mathrm{~g} \mathrm{~g}^{-1} ; \mathrm{pH}=7.3$, $T_{c}=60^{\circ} \mathrm{C}$ )

\section{THEORETICAI}

The problem will first be stated in general terms and will then be developed for some very simple reactions. A stirred tank reactor with a fixed volume $\left(V_{R}\right)$ is operated by constantly feeding two solutions, one containing the substrate and the other a soluble catalyst (for convenience, an enzyme). The global volumetric feed rate $(\dot{V})$ shall be constant and associated 
with the global concentrations of substrate $\left(S_{0}\right)$ and catalyst $\left(E_{0}\right)$. Under steady-state conditions the general mass balances for substrate and catalyst, the first in terms of substrate conversion $(X)$, may be given as follows:

$$
\begin{aligned}
X & =\frac{1}{S_{0}} r_{X} E_{A} \tau \\
E_{A} & =E_{0}-E_{D}=E_{0}-r_{D} \tau
\end{aligned}
$$

assuming a reaction kinetics of first order with respect to the catalyst concentration $\left(r_{V}=r_{X} E_{A}\right)$ and any kind of deactivation kinetics $\left(r_{D}\right)$. For convenience, the rate of deactivation shall be independent of substrate conversion. A compact description is obtained by simply substituting eq. (2) into eq. (1):

$$
X=\frac{1}{S_{0}} r_{X} \tau\left(E_{0}-r_{D} \tau\right) .
$$

Looking only for the local behaviour of the gradient of conversion as a function of space time by neglecting that both the rate of reaction and the rate of deactivation may be complex functions of conversion, space time, and catalyst concentration, the derivative may be simplified to yield

$$
\frac{\mathrm{d} X}{\mathrm{~d} \tau}=\frac{1}{S_{0}} r_{X}\left(E_{0}-2 r_{D} \tau\right) .
$$

This rudimentary analysis shows that the reaction kinetics is of much less importance than the deactivation kinetics with respect to the occurrence of a distinct maximum of conversion. It also suggests that such a phenomenon may only be observed for deactivation kinetics in which the expression in brackets of eq. (4) exactly vanishes. This is only the case for deactivation kinetics of less than first order. That this speculation is justified will be shown in terms of some simple examples.

For three examples, a reaction of first order with respect to both substrate and catalyst will be applied. The mass balance for the substrate may then be written in dimensionless form in terms of the degree of conversion:

$$
X=(1-X) \phi k_{X, 1} E_{0} \tau=(1-X) \phi D a_{X, 1}
$$

with $\phi$ the fraction of the residual activity of the catalyst and $D a_{X, 1}$ the Damköhler number of the first kind for a first-order catalytic reaction. The conversion is obtained from eq. (5)

$$
X=\frac{\phi D a_{X, 1}}{1+\phi D a_{X, 1}}
$$

as the classical solution for a first-order reaction taking place in a CSTR.

As the first case, deactivation kinetics of first order with respect to the catalyst concentration $\left(E_{A}\right)$ will be considered. The mass balance for a CSTR yields

$$
\phi=\frac{E_{A}}{E_{0}}=1-k_{D, 1} \phi \tau=1-\phi D a_{D, 1}
$$

with $D a_{D, 1}$ the Damköhler number of first kind for a first-order deactivation. The fraction of residual activity may then be obtained as

$$
\phi=\frac{1}{1+D a_{D, 1}} .
$$

Since in this simple case, both mass balances for substrate and catalyst are independent, the fraction of residual activity of eq. (8) may be substituted into the mass balance for the degree of conversion given by eq. (6) to yield the general solution:

$$
X=\frac{D a_{X, 1}}{1+\left(1+R_{1,1}\right) D a_{X, 1}}
$$

with

$$
R_{1,1}=\frac{D a_{D, 1}}{D a_{X, 1}}=\frac{k_{D, 1}}{k_{X, 1} E_{0}} .
$$

A simulation of this function for some different parameter values is given in Fig. 2. As expected from eq. (9), no distinct maximum of conversion is observed. The conversion tends towards a threshold value for the Damköhler number approaching infinity. The threshold value depends on the ratio of the Damköhler numbers of the deactivation and the catalytic reaction:

$$
X_{\lim }=\frac{1}{1+R_{1,1}} .
$$

For the second case discussed, the deactivation shall proceed according to a kinetics of zeroth order. The mass balance in terms of the fraction of residual activity is given by

$$
\phi=1-\frac{k_{D, 0} \tau}{E_{0}}=1-D a_{D, 0}
$$

Together with eq. (6) this leads to the global mass balance in term of conversion:

$$
\begin{array}{r}
X=\frac{\left(1-R_{0,1} D a_{X, 1}\right) D a_{X, 1}}{1+\left(1-R_{0,1} D a_{X, 1}\right) D a_{X, 1}} \\
\left(\text { for } R_{0,1} D a_{X, 1}<1\right)
\end{array}
$$

with

$$
R_{0,1}=\frac{D a_{D, 0}}{D a_{X, 1}}=\frac{k_{D, 0}}{k_{X, 1} E_{0}^{2}} .
$$

Results of a simulation are given in Fig. 3. It is clearly

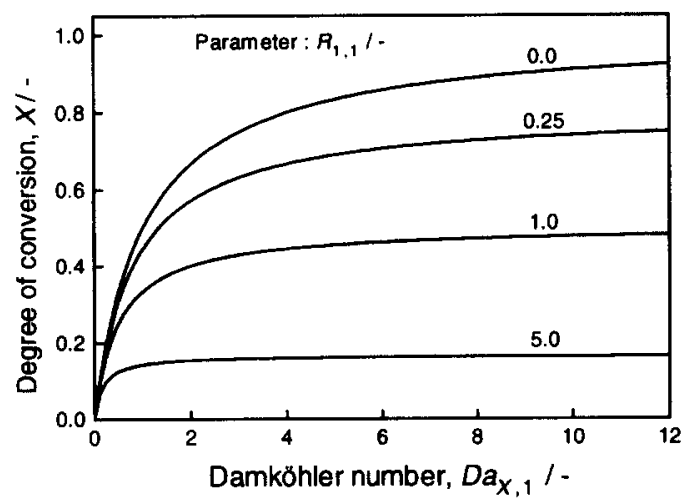

Fig. 2. Behaviour of a reaction system consisting of a catalytic reaction and catalyst deactivation both governed by first-order kinetics in a CSTR. 


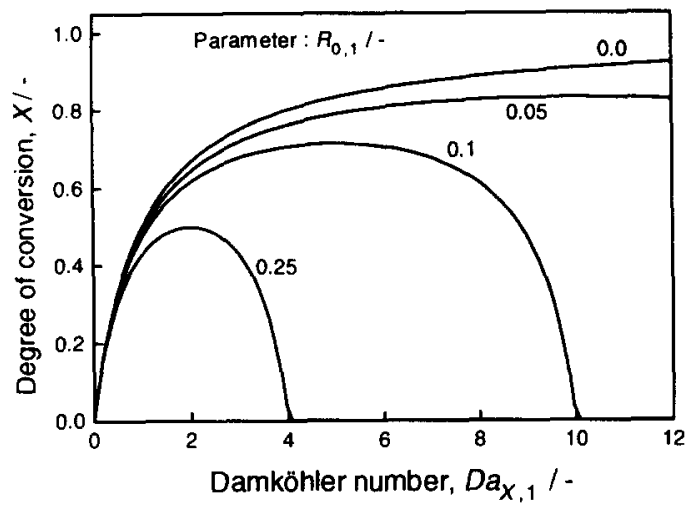

Fig. 3. Behaviour of a reaction system consisting of a catalytic first-order reaction with zeroth-order catalyst deactivation in a CSTR.

seen that distinct maxima of conversion appear. The analysis of eq. (12a) reveals that the conversion tends towards zero for the Damköhler number of deactivation approaching unity. The gradient of the degree of conversion with respect to space time shows that the maxima should occur at

$$
D a_{D, 0}^{\mathrm{opt}}=\frac{1}{2}, \quad D a_{X, 1}^{\mathrm{opt}}=\frac{1}{2 R_{0,1}}=\frac{k_{X, 1} E_{0}^{2}}{2 k_{p, 0}} .
$$

This implies that the maxima occur when half of the initial catalyst activity has undergone deactivation.

A third example is discussed, in which the deactivation proceeds with a kinetics of half-order. The mass balance in terms of the fraction of residual activity may be written as

$$
\phi=1-\phi^{1 / 2} \frac{k_{D, 1 / 2} \tau}{E_{0}^{1 / 2}}=1-\phi^{1 / 2} D a_{D, 1 / 2}
$$

This may be solved as

$$
\phi=\frac{1}{2}\left(2+R_{1 / 2,1}^{2} D a_{X, 1}^{2}\right)-\sqrt{\frac{1}{4}\left(2+R_{1 / 2,1}^{2} D a_{X, 1}^{2}\right)^{2}-1}
$$

with

$$
R_{1 / 2,1}=\frac{D a_{D, 1 / 2}}{D a_{X, 1}}=\frac{k_{D, 1 / 2}}{k_{X, 1} E_{0}^{3 / 2}}
$$

and $D a_{D, 1 / 2}$ the Damköhler number of the first kind for a half-order deactivation. Equation (15) may be combined with eq. (6) in order to obtain the global solution for the degree of conversion. Simulation results for this case are given in Fig. 4. The occurrence of distinct maxima of conversion as a function of the catalytic Damköhler number is evident. The analysis of eq. (15) together with eq. (6) reveals that the conversion tends to zero for the catalytic Damköhler number approaching infinity. The analysis of this model may be achieved as follows. From eq. (15) the Damköhler number of the catalytic reaction may be obtained as a function of the residual activity.

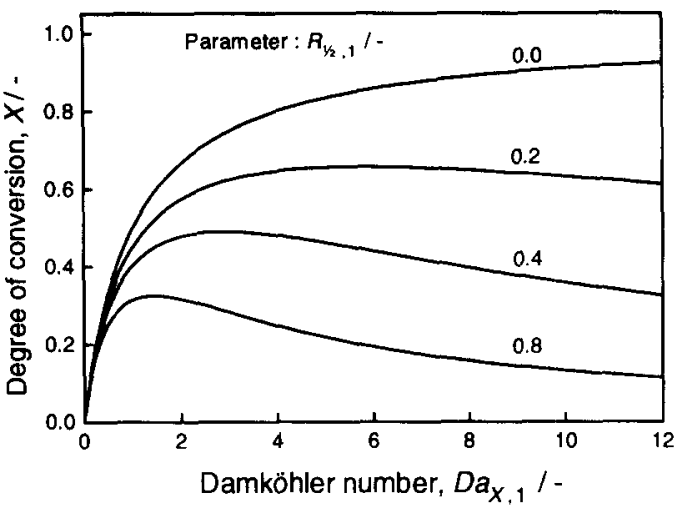

Fig. 4. Behaviour of a reaction system consisting of a catalytic first-order reaction with half-order catalyst deactivation in a CSTR.

Insertion of this expression into eq. (6) and evaluation of the derivative of the conversion with respect to the residual activity leads to the conditions for observing the distinct maxima:

$$
\begin{aligned}
\phi_{\mathrm{opt}} & =\frac{1}{3} \\
D a_{x, 1}^{\mathrm{opt}} & =\frac{1-\phi_{\mathrm{opt}}}{R_{1 / 2,1} \sqrt{\phi_{\mathrm{opt}}} .} .
\end{aligned}
$$

The examples discussed above represent rather simple reaction systems. However, the case studied by Margot (1992) represents a particular reaction because the enzyme considered is a protease (trypsin). Proteases may attack themselves due to their proteolytic activity. Enzymic catalysis is characterized by an adsorption/reaction mechanism with a kinetics following more or less the well-known Michaelis-Menten equation. However, the kinetics of autohydrolysis of proteases would follow kinetics with an order in between one and two depending on the saturation parameter of the protease with respect to itself. Therefore, there is no mechanistic argument for observation of a distinct maximum conversion, in this particular case.

The most likely situation for observation of the phenomenon discussed may, however, result when an enzymic reaction is performed in the presence of a protease which may deactivate the primary enzyme by proteolytic degradation. In this case, the deactivation of the primary enzyme would proceed with a Michaelis-Menten type of kinetics characterized by an apparent order of reaction in the range of zero and one. For convenience, an example will be discussed. The reaction system studied is given by

$$
\begin{gathered}
\mathrm{E}+\mathrm{S} \stackrel{K_{s, x}}{\longrightarrow} \mathrm{ES} \stackrel{k_{2, x}}{\longrightarrow} \mathrm{E}+\mathrm{P} \\
\mathrm{D}+\mathrm{E}_{A} \stackrel{K_{s, D}}{\longrightarrow} \mathrm{DE}_{A} \stackrel{k_{2, D}}{\longrightarrow} \mathrm{D}+\mathrm{E}_{D}
\end{gathered}
$$

with $D$ denoting the protease, $E_{A}$ the remaining active enzyme species ( $E$ and $E S$ ), and $E_{D}$ the denatured enzyme. The global balance is simplified by assuming equilibrium for formation of the active complexes, ES 
and $\mathrm{DE}_{A}$, and that the amount of protease present may be much less than the substrate transforming enzyme. The latter condition implies that the concentration of active complex $\mathrm{DE}_{A}$ may be neglected in the balance for the substrate transforming enzyme (E). By introducing the following definitions:

$$
\begin{gathered}
E+E S=\phi E_{0} \\
E_{D}=(1-\phi) E_{0} \\
k_{X, 1}=\frac{k_{2, X}}{K_{s, X}} \\
k_{D, 1}=\frac{k_{2, D}}{K_{s, D}} \\
s_{0 s}=\frac{S_{0}}{K_{s, X}} \\
e_{0 s}=\frac{E_{0}}{K_{s, D}} \\
D a_{X, 1}=k_{X, 1} E_{0} \tau \\
D a_{D, 1}=k_{D, 1} D_{0} \tau
\end{gathered}
$$

the global balance for continuous stirred tank reactor operation is given by

$$
\begin{aligned}
\phi= & \frac{1}{2}\left[1-\frac{1+R_{1,1} D a_{X, 1}}{e_{0 s}}\right] \\
& +\sqrt{\frac{1}{4}\left[1-\frac{1+R_{1,1} D a_{X, 1}}{e_{0 s}}\right]^{2}+\frac{1}{e_{0 s}}} \\
X= & \frac{1}{2}\left[1+\frac{1+\phi D a_{X, 1}}{s_{0 s}}\right] \\
& -\sqrt{\frac{1}{4}\left[1+\frac{1+\phi D a_{X, 1}}{s_{0 s}}\right]^{2}-\frac{\phi D a_{X, 1}}{s_{0 s}}}
\end{aligned}
$$

with

$$
R_{1,1}=\frac{D a_{D, 1}}{D a_{X, 1}}=\frac{k_{2, D} D_{0}}{k_{2, X} E_{0}} \frac{K_{s, X}}{K_{s, D}} .
$$

Characteristic results of the simulation of this model are shown in Fig. 5. The reaction conditions have been chosen in order to attain a proteolytic deactivation of the substrate transforming enzyme with an order of reaction close to zero under initial conditions.

\section{DISCUSSION}

It is shown that the conversion-space time profile for continuous stirred tank reactor operation may exhibit a distinct maximum of conversion in the case of catalyst deactivation proceeding with a kinetics of less than first order. Different kinetic models for catalytic transformations as well as simple models for catalyst deactivation have been studied theoretically, but no other combination showed a distinct maximum in conversion. The only enzyme known for

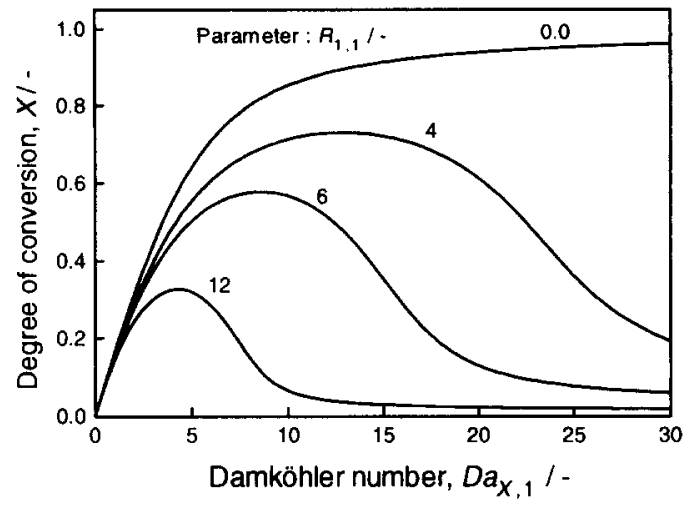

Fig. 5. Behaviour of a reaction system consisting of an enzymic reaction with protease degradation of the enzyme in a CSTR (parameters: $s_{0 s}=5 ; e_{0 s}=100$ ).

which a deactivation kinetics of less than first order has been postulated is the lactase of Aspergillus oryzae which undergoes deactivation by a formal kinetics of half-order according to a report of Flaschel et al. (1985). However, this kinetic model has been obtained for discontinuous reactor operation, and since a halforder kinetics would hide a more complex reaction mechanism, a verification in a continuous stirred tank reactor appears to be unlikely. Therefore, a simple catalyst deactivation mechanism showing the observed phenomenon may not exist.

The occurrence of a distinct maximum in the conversion-space time profile as experimentally observed by Margot (1992) remains unexplained. A detailed analysis of the deactivation of trypsin during discontinuous reactor operation revealed a series type inactivation mechanism (Margot et al., 1992) which should not lead to a distinct conversion maximum for CSTR operation. More complex deactivation kinetics like those which may lead to steady-state multiplicities in CSTRs (Patnaik, 1993) have not been investigated so far.

The most likely situation for a maximum conversion to occur in continuous stirred tank operation has been demonstrated to show up in the case of an enzymic reaction accompanied by a proteolytic degradation of the substrate transforming enzyme due to the presence of a protease. Such reaction systems are of practical importance, because most of the enzymes produced for industrial application are used in soluble form without recovery. The presence of proteases in crude enzyme preparations is of primary concern as well (Sproull et al., 1976). However, the discussion has shown that the appearance of a distinct maximum in the conversion-space time profile for catalytic reactions in continuous stirred tank reactors may not have been observed so far, because the phenomenon seems to be rare on theoretical reasoning and, in addition, continuous stirred tank reactors are rarely applied for experimental studies of enzymic reactions. 


\section{NOTATION}

$D a_{n} \quad$ Damköhler number of the first kind, approximation for an $n$th order reaction, dimensionless

$D \quad$ protease concentration, $\mathrm{g}^{-1}$

$E$ concentration of free active enzyme (catalyst) species, $\mathrm{g}^{-1}$

$E_{A}$ total concentration of active enzyme (catalyst) species, $\mathrm{g}^{-1}$

$E_{D} \quad$ concentration of inactivated enzyme (catalyst) species, $\mathrm{g} \mathrm{I}^{-1}$

ES concentration of enzyme-substrate complex, $\mathrm{g}^{-1}$

$e_{0 s} \quad$ saturation parameter for proteolytic enzyme degradation, dimensionless

$k_{2} \quad$ rate constant of enzymic reactions (variable)

$k_{n} \quad$ rate constant of $n$th order kinetics (variable)

$K_{s} \quad$ Michaelis-Menten constant (variable)

NPN "non-protein nitrogen" or fraction of solubilized protein, dimensionless

$R_{n, m} \quad$ ratio of the Damköhler number of deactivation of $n$th order and the Damköhler number of the catalytic reaction of $m$ th order

$r_{D} \quad$ kinetic expression for the (volumetric) rate of catalyst deactivation, $\mathrm{g} \mathrm{l}^{-1} \mathrm{~min}^{-1}$

$r_{V} \quad$ volumetric rate of the catalytic reaction, $\operatorname{mol~}^{-1} \min ^{-1}$

$r_{\boldsymbol{X}} \quad$ kinetic expression for the rate of substrate consumption, $\mathrm{mol} \mathrm{g}^{-1} \mathrm{~min}^{-1}$

$S \quad$ substrate concentration, $\mathrm{mol} \mathrm{l}^{-1}$

$T_{c} \quad$ operating temperature, ${ }^{\circ} \mathrm{C}$

$\dot{V} \quad$ volumetric flow rate of reactor feed, $1 \mathrm{~min}^{-1}$

$V_{R} \quad$ reactor volume, 1

$X$ degree of substrate conversion, dimensionless

\section{Greek letters}

$\phi \quad$ fraction of residual enzyme (catalyst) activity, dimensionless $\tau \quad$ space time in a continuous stirred tank reactor $(\mathrm{CSTR})\left(=V_{R} / \dot{V}\right)$, min

\section{Subscripts and superscripts}

D refers to deactivation reaction

lim limiting quantity for high space times

opt refers to operating conditions for which a distinct maximum conversion is observed

$X \quad$ refers to enzymic (catalytic) reaction

0 refers to conditions in the reactor feed solution

\section{Abbreviations}

CSTR continuously operated ideal stirred tank reactor

D enzyme (catalyst) deactivating protease

$\mathrm{DE}_{A}$ enzyme-protease complex

$\mathrm{E}$ free (dissociated) enzyme (catalyst), active form

$\mathrm{E}_{A}$ total enzyme (catalyst) $(\mathrm{E}+\mathrm{ES})$, active form

$E_{D} \quad$ enzyme (catalyst), inactive (denatured) form

ES enzyme-substrate complex

$P \quad$ product

S substrate

\section{REFERENCES}

Flaschel, E., van Griethuysen, E. and Renken, A., 1985, Deactivation kinetics and temperature optimization of enzymatic batch processes. Ger. Chem. Engng 8, 362-371 Margot, A., 1992, Développement d'un réacteur continu pour l'hydrolyse enzymatique de protéines du lactosérum. Ph.D. thesis No. 994, Swiss Federal Institute of Technology Lausanne

Margot, A., Richoz, C., Flaschel, E. and Renken, A., 1992, Einfluß der Trypsin-Desaktivierung auf die Hydrolyse von Molkeproteinen. Chemie.-Ingr.-Tech. 64, 551-553.

Patnaik, P. R., 1993, Steady-state multiplicity in enzyme deactivation: application of a simple graph theoretic method. Enzyme Microb. Technol. 15, 162-166.

Sproull, R. D., Lim, H. C. and Schneider, D. R., 1976, A model for enzymatic isomerization of D-glucose to D-fructose in a batch reactor. Biotechnol. Bioeng. 18 , 633-648. 